\title{
Identifying Microdeletion Syndromes in Patients with Intellectual Disability Using Molecular Genetic Testing: An Example for the Brazilian Public Health Care System
}

\author{
Adriana Rosolio Costa Sabbag ${ }^{1}$, Bruno Garcia Rocha ${ }^{1}$, Lucimar Retto da Silva de Avó ${ }^{2}$, Carla Maria Ramos \\ Germano $^{1,2}$, Euclides Mathe ucci Junior ${ }^{1}$, Débora Gus mão Melo ${ }^{1,2, *}$ \\ ${ }^{1}$ Postgraduate Program in Biotechnology, Federal University of São Carlos (UFSCar), São Carlos, SP, Brazil \\ ${ }^{2}$ Department of Medicine, UFSCar, São Carlos, SP, Brazil \\ ${ }^{3}$ Dep artment of Chemistry, UFSCar, São Carlos, SP, Brazil \\ *Corresponding author: dgmelo@ufscar.br
}

Received April 17, 2013; Revised May 17, 2013; Accepted May 18, 2013

\begin{abstract}
Unexplained intellectual disability is a clinical situation in which molecular diagnostic techniques should be indicated. The diagnostic yield of Multiplex Ligation-dependent Probe Amplification (MLPA) in a cohort of patients with intellectual disability and dysmorphic features was investigated to identify microdeletion syndromes. We aimed to provide an example of the utilization of MLPA method in the medical care routine that can be useful for planning the inclusion of molecular genetic testing in the Brazilian public health care system. This study was based on 57 patients who had different degrees of intellectual disability with etiology not determined. All patients had normal brain CT scan or MRI and normal karyotype, and patients with environmental damage history were not included. Two of the 57 patients were referred to molecular genetic testing as they were clinically diagnosed as having Williams syndrome. MLPA test costs were calculated (human resources and equipment costs were not included in the calculation). MLPA revealed chromosomal imbalance in 4 out of 57 patients (7\%). These imbalances were associated with well-described microdeletion syndromes: 3 patients had Williams syndrome ( 1 without clinical diagnosis) and 1 patient had 22q11.21 deletion syndrome. The MLPA analysis cost per individual, considering DNA extraction and laboratory reagents, was US\$66.40. In this study, MLPA confirmed its value as a promising technique as it has adequate feasible characteristics to identify microdeletion syndromes in patients who had unexplained intellectual disability. This work suggests that MLPA can be a viable alternative to implement molecular genetic testing in the Brazilian public health care system, considering its cost-effectiveness.
\end{abstract}

Keywords: biomedical technology assessment, molecular diagnostic techniques, genetic testing, genetic services, intellectual disability, public health, brazil

\section{Introduction}

Molecular genetic testing have been developed for more than 2,900 diseases, of which about 2,700 are currently available for use in clinical settings [1]. Multiple congenital anomalies, autis $\mathrm{m}$ spectrum disorders and unexplained developmental delay/intellectual disability are clinical situations in which molecular diagnostic techniques should be indicated [2,3].

Intellectual disability (ID) is characterized by significant limitations in both intellectual functioning and adaptive behavior that begin before the age of 18 years [4], occurring in $1-3 \%$ of the general population $[5,6,7,8]$. A diagnosis of ID is usually made when IQ testing reveals an IQ below 70, which means that the diagnosis is frequently not confirmed until late childhood or early adulthood. However, most people with ID are identified early in childhood on the basis of concern about global developmental delays, which may include motor, cognitive, and speech delays [3]. The term global developmental delay is usually reserved for younger children (i.e., typically less than 5 years old, when available valid instruments for assessing intelligence are not generally applicable), whereas the term ID is usually applied to older children when IQ testing is more validated and reliable [5,9].

ID represents one of the most frequently diagnosed disabling condition in our society, and a life long disability characterized by impairment of cognitive and adaptive skills [10]. Everyone who has ID or children who have been diagnosed with global developmental delay should have a comprehensive evaluation to establish the etiology of the disability. ID etiology is tremendously heterogeneous and, unfortunately, in about one-half of the cases the cause of ID is still elusive [7,10,11]. Anything that damages and interferes with the growth and maturation of the brain can lead to ID, and this might happen before, during or after children's birth. Moreover, genetically determined ID etiology (comprising chromosomal aberrations, single-gene disorders, and other genetic conditions) account for 17 to $41 \%$ of cases, depending on patient selection [11], inclusion or not of 
Down syndrome in the cohort [7] and the different techniques that have been selected for analysis $[5,10,12]$.

The ID etiologic diagnosis has immediate implications with respect to recurrence risks and therapeutic imperatives, possessing the potential to modify management and expected outcomes [9]. Future medical challenges and prognosis for disabled children can be more accurately addressed in a child with a known diagnosis [5,9]. The family of a child with global developmental delays or ID often experiences the feeling of hopelessness and frustration, and an etiologic diagnosis can help the family to cope $[13,14,15]$. In addition, a specific etiologic diagnosis can make social support and information more accessible for families and professionals. For many diagnoses, specific management guidelines are now available [16]. For primary care providers and families, there are specific benefits in establishing an etiologic diagnosis including clarification of etiology, prognosis, genetic mechanis $\mathrm{m}(\mathrm{s})$, recurrence risks, and treatment options; avoidance of unnecessary tests; information regarding management or surveillance and family support; access to research and treatment protocols. There is also the opportunity for management of selected patients in the context of a nurse care home facility to ensure better health and social outcomes for the disabled child [9].

In medical check-ups, patients who have unexplained developmental delay or ID are subjected to a variety of diagnostic tests, including physical and dysmorphologic examination, metabolic and neurological investigations, neuroimaging, and genetic testing [3,5,7,11,17]. Cytogenetic abnormalities are considered to represent a major cause of ID and a huge amount of data correlating specific unbalanced chromosomal imbalances to clinical phenotypes, in developmental delay or ID patients, have been compiled [7]. Detecting chromosomal aberrations by karyotyping is limited by the resolution of the chromosome banding pattern. This implies that gains and losses of less than $5-10 \mathrm{Mb}$ may go undetected in karyograms having a resolution of 550 bands, accepted as the standard [18]. A substantial proportion of syndromes associated with ID are caused by chromosomal microdeletions and microduplications smaller than $3-5 \mathrm{Mb}$ $[10,19]$. Parallel to the advent of novel molecular diagnostic techniques, several new cryptic chromosomal aberrations have been discovered over the last few years and a consistent number of ID cases, previously considered idiopathic, are now classified as syndromic conditions with clinical recognizable phenotypes [10,19,20,21]. Although some of these micro-deletions/duplications result in a specific phenotype leading to a clinical diagnosis, a number of patients who have wellknown syndromes are in fact not diagnosed clinically. In some cases, a diagnosis may be reached by applying a whole genome screening technique, such as array-CGH and higher-density platforms, such as SNP-array [3,10]. A considerably faster and more cost-effective alternative to detect a cluster of ID syndromes is the Multiplex Ligationdependent Probe Amplification technique [22,23]. The Multiplex Ligation-dependent Probe Amplification (MLPA) was first described in 2002 and is a variation of the multiplex polymerase chain reaction that permits multiple targets (up to 50) to be amplified with only a single primer pair. The MLPA technique is easy to use and can be performed in many laboratories, as it only requires a thermocycler and a capillary electrophoresis equipment [24].

Using molecular biology techniques such as MLPA, fluorescence in situ hybridization (FISH) and wholegenome array screening (WGAS), the detection rate of chromosomal abnormalities in ID patients can be substantially increased [25,26,27]. WGAS alone detects $99 \%$ of all chromosomal alterations, and recent reports have suggested that array-CGH should replace conventional karyotyping as the first diagnostic test used to detect chromosomal abnormalities in ID patients $[2,3,7,28,29]$. However, array techniques are relatively expensive and laborious, they often require considerable follow-up parental investigations and they also detect a substantial number of imbalances of unknown significance, which may hinder the correct interpretation of laboratory results and require clinical expertise to correlate genotypephenotype properly [10,22]. Additionally, the high cost of array-CGH makes it unfeasible for many developing countries, such as Brazil [23]. FISH represented an important advance towards a reliable detection of smaller chromosome rearrangements and allowed physicians to rapidly confirm the diagnosis of a previously suspected micro-deletion/-duplication syndrome and to examine the subtelomeres region of each chromosome. FISH analys is is a locus-specific probe and only a limited number of specified regions of the genome can be simultaneously investigated, restricting its routine application [7,30]. In this context, MLPA has emerged as a promising technique to detect chromosomal abnormalities in many clinical conditions [23,31].

MLPA is a multiplex PCR method able to detect abnormal copy numbers of up to 50 different genomic DNA sequences simultaneously. Although MLPA is not suitable for WGAS, it is a good alternative to array-based techniques for many routine applications [22,23]. MLPA is a relatively fast and ine xpensive method, and more than 300 probe sets now commercially available are designed to diagnose either relatively common or very rare diseases. Using MLPA to identify the most common microdeletion/-duplication syndromes represents a reasonable choice when designing a testing algorithm for ID [22], even in developing countries where resources are limited [23].

We investigated the diagnostic yield of MLPA in a cohort of patients who have unexplained intellectual disability and dysmorphic features to identify microdeletion syndromes. The objective of this study was to provide an example of the utilization of MLPA method in the medical care routine that can be useful for planning the inclusion of molecular genetic testing in the Brazilian public health care system.

\section{Materials and Methods}

\subsection{Patients}

This study is a cross-sectional descriptive-analytical research conducted in a genetic service of a medical school, inserted in the Brazilian public health care system, and located in the city of São Carlos, in São Paulo State, Brazil southeastern. The city has about 220,000 
inhabitants and a human development index of 0.841 [32]. It is a regional polo, whose economy is based on agricultural and industrial activities. The city has public services, including health services, which attend the small towns around. Two public universities and two research centers of the Brazilian Agricultural Research Corporation are located in São Carlos, reinforcing its vocation as a center of regional scientific and technological development.

This study was approved by the Human Research Ethics Committee from the Federal University of São Carlos $\left(\mathrm{N}^{\mathrm{O}}\right.$ 253/2010). The research was based on 57 patients of both sexes who had different degrees of ID or global developmental delay and dysmorphic features. The Table 1 shows patients clinical features.

Table 1. Patients clinical features

\begin{tabular}{|c|c|}
\hline \multicolumn{2}{|c|}{ Sex } \\
\hline Male, $\mathrm{n}(\%)$ & $33(57.9)$ \\
\hline Female, n (\%) & $24(42.1)$ \\
\hline Mean age $\pm \mathrm{SD}, \mathrm{y}$ (minimum - maximum) & $12.4 \pm 7.3(1.4-36)$ \\
\hline Male mean age \pm SD, y (minimum - maximum) & $12.5 \pm 7.3(1.4-36)$ \\
\hline Female mean age \pm SD, y (minimum - maximum) & $12.3 \pm 7.5(3.1-32.4)$ \\
\hline Global developmental delay, $\mathrm{n}(\%)$ & $11(19.3)$ \\
\hline Intellect ual disability, $\mathrm{n}(\%)$ & $46(80.7)$ \\
\hline Mild, $\mathrm{n}(\%)$ & $5(8.8)$ \\
\hline Moderate, $\mathrm{n}(\%)$ & $29(50.9)$ \\
\hline Severe or profound, $\mathrm{n}(\%)$ & $12(21)$ \\
\hline \multicolumn{2}{|c|}{ Malformations associated } \\
\hline Congenital heart disease, $\mathrm{n}(\%)$ & $8(14)$ \\
\hline Microcephaly, $\mathrm{n}(\%)$ & $4(7)$ \\
\hline Macrocephaly, $\mathrm{n}(\%)$ & $1(1.75)$ \\
\hline \multicolumn{2}{|c|}{ Others clinical conditions associat ed } \\
\hline Obesity, n (\%) & $4(7)$ \\
\hline Short stature, $(\%)$ & $2(3.5)$ \\
\hline Epilepsy, n (\%) & $2(3.5)$ \\
\hline
\end{tabular}

A medical geneticist (DGM) evaluated all patients prior to referral to the study and a detailed pre-, peri- and postnatal history was collected; patients with a history of pregnancy/birth complications, toxic exposition, malnutrition, trauma, infections and understimulation were not included. All patients had normal brain CT scan or MRI and normal karyotype. Two of the 57 patients were referred to molecular genetic testing as they were thought to have Williams syndrome.

\subsection{Genetic Testing}

Karyotyping, with G-banding analysis, were performed on methaphase preparations of peripheral blood ly mphocytes, using standard techniques and a resolution of $400-550$ bands.

MLPA is based on the analysis of relative changes in probe signals. Thus, a given sample will not provide the information needed to estimate copy number changes without a reference sample to compare it to. Reference samples are samples in which the target sequences are assumed to have a 'normal' copy number. Thus, for molecular genetic testing we selected 5 healthy subjects who provided the reference samples. The genomic DNA were extracted from peripheral blood leukocytes using the QIAamp DNA Blood Kit (Qiagen $\left.{ }^{\circledR}\right)$, according to the manufacturer's instructions.

All participants were tested using a MRS-MLPA P064 probe set (MRC-Holland, Amsterdam, The Netherlands), which can detect microdeletions associated with: 1p36 deletion syndrome, Williams syndrome, Smith-Magenis syndrome, Miller-Dieker syndrome, 22q11.21 deletion syndrome, Prader-Willi syndrome, Angelman syndrome, Alagille syndrome, Saethre-Chotzen syndrome and Sotos syndrome. Some of these syndromes are not always easily diagnosed by physical examinations, as not all the common clinical findings associated with a particular syndrome are present in a patient or they vary among patients from different ethnics groups.

MLPA was carried out according to the manufacturer's instructions, with 5 reference samples in each MLPA run. Electrophoresis and fragment analysis were performed using the Mega-BACE TM 1000 DNA analysis system and Fragment Profiler software version 1.2 (GE Healthcare). The data were analyzed using GeneMarker software (Softgenetics ${ }^{\circledR}$ ) and Coffalyser software (MRC Coffalyser(). Results were considered not normal when the relative peak height ratio was below 0.75 (microdeletion) or above 1.25 (mic roduplication).

\subsection{Cost Analysis}

The costs for performing the MLPA test were calculated including only laboratory reagents and kits, considering prices for the purchase in the domestic market and for transportation. Costs were initially calculated in local currency, Brazilian real (RS\$), and then converted to US dollars (US\$) using the day's exchange rate (US\$ 1.00 $=\mathrm{RS} \$ 2.03)$.

\section{Results}

MLPA revealed chromosomal imbalances in 4 out of 57 patients $(7 \%)$. These imbalances were associated with well-described microdeletion syndromes: 3 patients had Williams syndrome and 1 patient had 22q11.21 deletion syndrome (Figure 1).

The cost for MLPA analysis per individual, considering DNA extraction and laboratory reagents was US\$ 66.40. Hu man resources and equipment costs were not included. 

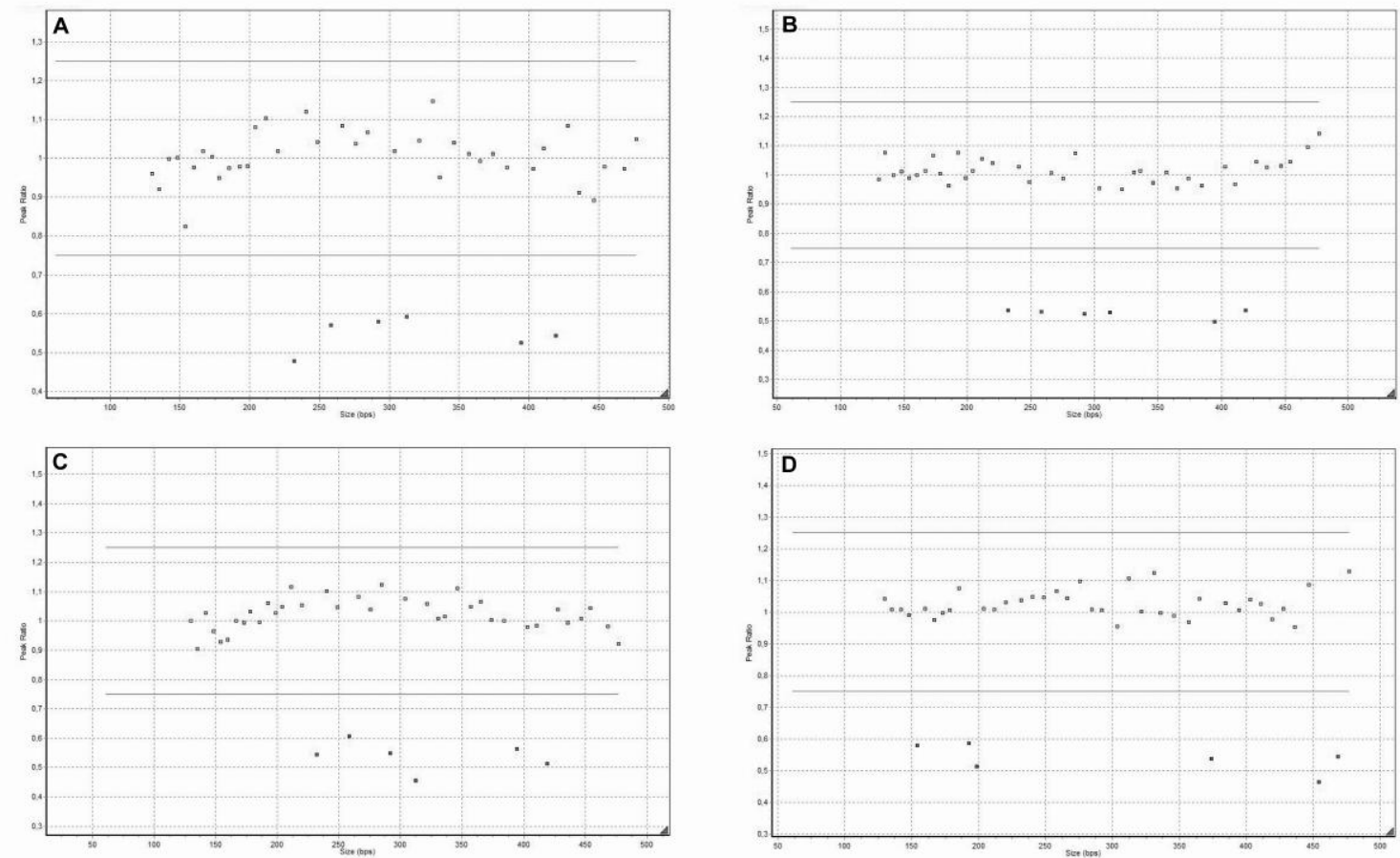

Figure 1. MLPA results, with kit MRS-MLPA P064 analyzed by GeneMarker software, showing abnormal findings for the six probes at $7 \mathrm{q} 11.23$ (Williams syndrome-A, B and C) and 22q13.3 (D)

\section{Discussion}

In this study, we evaluated the ability of MLPA to detect common microdeletion syndromes associated with ID and dysmorphic features in patients with normal conventional karyotype and we found a diagnostic rate of $7 \%$. The clinical findings of the patients were compatible with the microdeletions detected. Two microdeletions were found in patients who were thought to have a specific syndrome at referral (Williams syndrome). However, two other imbalances (including $1 \mathrm{~W}$ illiams syndrome and 1 22q11.21 deletion syndrome) were detected among patients, where no specific suspicion had been reported at referral to molecular genetic testing. The fact that the specific syndromes were not suspected clinically may reflect the heterogeneity in syndromes' phenotypes [33,34] and reinforces the requirement of a feasible method to search for the most common microdeletion syndromes in patients with unexplained ID and dysmorphic features.

For a better cost-effectiveness of the molecular genetic testing, the best MLPA-kit should be selected according to patients' phenotype. For this, in our experience was important to establish a good communication between the physician and the diagnostic laboratory. Moreover, we recommend careful analysis of each patient, particularly when no other confirmatory test could be provided. If possible, MLPA probe results should be confirmed by another method, as Southern blots, long range PCR and/or FISH, which was not done in this study. In doubtful cases, normal MLPA results should be followed up by a highresolution microarray platform analys is.

A health system should have a healthcare plan for patients with ID, including appropriate etiologic investigation and genetic counseling. Brazil is the fifth most populous country in the world, and has more than 186 million inhabitants. The Brazilian public health care system, called the Unified National Health System (in Portuguese, "Sistema Ún ico de Saúde" - SUS), covers the medical expenses for $75 \%$ of the population. The SUS was implemented in 1990, based on three ideological principles - universality, comprehensiveness and equity, together with four organizing principles - hierarchy, decentralization, regionalization and popular participation [35]. Despite SUS's widespread coverage, access to genetic evaluation, testing and counseling are restricted to research facilities or university hospitals. This situation is similar to many other developing nations and reflects a dearth of clinical geneticists and genetic services: in Brazil, the geographical spread of genetics professionals and services correlates to population density and human development index; so in the more remote, poorer and less densely populated areas (particularly in the North and Northeast) there is a great shortage of professionals and laboratories [31,36,37,38,39].

In January 2009, the Brazilian Ministry of Health introduced, through Ordinance No. 81, the "National Policy for Integral Attention in Clinical Genetics in SUS". The aim of this policy was to structure a network of services, hierarchy and regionalization to allow comprehensive care in clinical genetics, and to improve access to this specialized field by the general population [40]. A working group has been summoned by the Ministry of Health to study how to implement this Policy in SUS, securing access to genetic evaluation, testing and counselling.

In Brazil, kariotype is the only genetic testing currently available in the SUS, which has payment coverage limited to US\$16.0 for each test. Private laboratories provide karyotyping, FISH, and others molecular analyses for 
various genetic diseases, but only a minority of the Brazilian population can afford private health insurance or direct payment for genetic testing. An experience of a reference center, describing the diagnosis of $22 \mathrm{q} 11.2$ deletion suggests that the implementation of FISH or MLPA would increase the costs of the testing by more than two or three times when compared with karyotyping [31]. These results are similar to our results: the cost of genetic testing for etiological investigation increased 4.15 times when we included the MLPA test to identify microdeletion syndromes in patients with unexplained ID.

The laboratory equipment and human resources required are important points to consider to implement diagnostic tests. The strategy adopted in this study was to use the infrastructure that already existed in a genetic service linked to a University. Most of the public medical genetic services in Brazil are linked to universities and research centers which have permitted public access to genetic services of quality in some regions of the country [31,38]. Moreover, using research grants to implement new technology for molecular analyses has permitted the ID etiology diagnosis in patients enrolled in research projects, like this one [23,31]. However, genetic testing diagnosing well-known syndromes should not be considered as research anymore, and the costs must be transferred from research centers to the public health care system.

The association between University and SUS could lead to a good use of the resources already established and calls for the implementation of genetic testing on the public health care system [31]. For these reasons, the strategy herein proposed utilized the structure of a University, reducing the need for equipment investment. Human resources involved in this study were also from the Institution as the researchers were postgraduate students. For a large-scale implementation, the SUS would have to invest in its own specialized human resources.

The lack of human resources (clinical and laboratorial) is an impeditive factor to implement the National Policy for Integral Attention in Clinical Genetics in SUS and to insert genetic testing in Brazil. The survey data "Medical Demography in Brazil", conducted by the Federal Council of Medicine and published in December 2011 indicates the existence of 156 medical geneticists distributed over the country [41]. One of the challenges faced is how to promote education, training and a fair distribution of human and physical resources (laboratory and clinical geneticists, as well as genetic counsellors and nurses); thereby expanding SUS users access to an appropriate and useful genetic testing, by adding effective health technology assessment, ethical meaning and social responsibility to the provision of medical genetics services in Brazil [39].

A report about public clinical genetic policies in Brazil pointed out that, to establish a functional and economic laboratory network, the implementation of specialized laboratories in all genetic services or even in all 26 Brazilian States would not be feasible [31]. We agree with other authors [31,36] who believe that basic laboratories, such as cytogenetic laboratories, could be implemented regionally or at a State level, and more specialized analyses could be offered by a few reference centers in genetics around the country.
The present study offers a portrayal of a common situation in small cities of Brazil and the solution proposed could be used to start planning a national approach for genetic testing in the public health care system. Perhaps, a good strategy to implement genetic testing is to use the infrastructure and human resources that already existed in genetics services linked to universities and research centers, concentrating on specific diagnosis and reducing costs of molecular genetic testing.

\section{Conclusions}

Although the knowledge of the cause of ID usually does not allow a treatment, it is helpful for disease management by parents, teachers and health professionals, as well as participation in support groups. Additionally, etiologic diagnosis provides a significant and long-lasting emotional relief for parents. In this study, MLPA emerged as a technique with adequately robust characteristics and is relatively easy to use to identify microdeletion syndromes in patients with unexplained ID. This work suggests that MLPA can be a viable alternative to implement molecular genetic testing in the Brazilian public health care system, considering its costeffectiveness.

\section{Acknowledgements}

We gratefully acknowledge the patients and their parents. This study was supported by funding from CAPES (Brazilian Coordination for the Improvement of Higher Education Personnel) - Program NANOBIOTEC 856/2009, CNPq (National Counsel of Technological and Scientific Development) and FAPESP (São Paulo Research Foundation), process 2010/16613-3.

\section{Statement of Competing Interests}

The authors have no competing interests.

\section{List of Abbreviations}

MLPA=Multiplex Ligation-dependent Probe Amplification; $\mathrm{CGH}=$ Comparative Genomic Hybridization; SNP = Single Nucleotide Polymorphism; FISH = Fluorescence In Situ Hybridization; WGAS = Whole Genome Array Screening; SUS = Unified National Health System (in Portuguese, "Sistema Único de Saúde").

\section{References}

[1] GeneTests Medical Genetics Information Resource [Online]. Copyright, University of Washington, Seattle. 1993-2013.

[2] Miller, D.T., Adam, M.P., Aradhya, S., et al. "Consensus statement: chromosomal microarray is a first-tier clinical diagnostic test for individuals with developmental disabilities or congenital anomalies", Am J Hum Genet, 86(5), 749-764, May 2010.

[3] Mefford, H.C., Batshaw, M.L. and Hoffman, E.P., "Genomics, intellectual disability, and autism", NEngl J Med, 366(8), 733-743, Feb. 2012. 
[4] American Psychiatric Association, Diagnostic and Statistical Manual of Mental Disorders-DSM-IV-TR. Washington, DC, American Psychiatric Association, 2000, 41-46.

[5] Shevell, M., Ashwal, S., Donley, D., et al. Quality Standards Subcommittee of the American Academy of Neurology and Practice Committee of the Child Neurology Society, "Practice parameter: evaluation of the child with global developmental delay: report of the Quality Standards Subcommittee of the American Academy of Neurology and The Practice Committee of the Child Neurology Society", Neurology, 60(3), 367-380, Feb. 2003.

[6] Stegmann, A.P., Jonker, L.M. and Engelen, J.J., "Prospective screening of patients with unexplained mental retardation using subtelomeric MLPA strongly increases the detection rate of cryptic unbalanced chromosomal rearrangements", Eur J Med Genet, 51(2), 93-105, Mar-Apr. 2008.

[7] Hochstenbach, R., van Binsbergen, E., Engelen, J., et al. “Array analysis and karyotyping: workflow consequences based on a retrospective study of 36,325 patients with idiopathic develop.mental delay in the Netherlands", Eur J Med Genet, 52(4), 161-169, Jul-Aug. 2009

[8] Vissers, L.E., de Vries, B.B. and Veltman, J.A. "Genomic microarrays in mental retardation: from copy number variation to gene, from research to diagnosis", J Med Genet, 47(5), 289-297, May 2010.

[9] Moeschler, J.B., "Genetic evaluation of intellectual disabilities", Sem in Pediatr Neurol, 15(1), 2-9, Mar. 2008.

[10] Galasso, C., Lo-Castro, A., El-Malhany, N. and Curatolo, P. "Idiopathic mental retardation and new chromosomal abnormalities", Ital J Pediatr, 36, 17, Feb. 2010.

[11] Rauch, A., Hoyer, J., Guth, S., et al. "Diagnostic yield of various genetic approaches in patients with unexplained developmental delay or mental retardation", Am J Med Genet A, 140(19), 20632074, Oct. 2006.

[12] Bernardini, L., Alesi, V., Loddo, S., et al. "High-resolution SNP arrays in mental retardation diagnostics: how much do we gain?" Eur J Hum Genet, 18(2), 178-185, Feb. 2010.

[13] Makela, N.L., Birch, P.H., Friedman, J.M. and Marra, C.A., "Parental perceived value of a diagnosis for intellectual disability (ID): a qualitative comparison of families with and without a diagnosis for their child's ID", Am J Med Genet A, 149A (11), 2393-2402, Nov. 2009.

[14] Lewis, C., Skirton, H. and Jones, R. "Living without a diagnosis: the parental experience", Genet Test Mol Biomarkers, 14(6), 807815, Dec. 2010.

[15] Madeo, A.C., O'Brien, K.E., Bernhardt, B.A. and Biesecker, B.B., "Factors associated with perceived uncertainty among parents of children with undiagnosed medical conditions", Am J Med Genet A, 158A (8), 1877-1884, Aug. 2012.

[16] Cassidy, S.B. and Allanson, J. (eds.), Management of Genetic Syndromes, $2^{\text {nd }}$ ed. Hoboken, NJ, John Wiley \& Sons, 2005.

[17] Van Karnebeek, C.D., Jansweijer, M.C., Leenders, A.G., Offringa, M. and Hennekam, R.C., "Diagnostic investigations in individuals with mental retardation: a systematic literature review of their usefulness", Eur J Hum Genet, 13(1), 6-25, Jan. 2005.

[18] Hastings, R.J., Cavani, S., Bricarelli, F.D., Patsalis, P.C., Kristoffersson, U. and ECA PWG Co-ordinators, "Cytogenetic Guidelines and Quality Assurance: a common European framework for quality assessment for constitutional and acquired cytogenetic investigations", Eur J Hum Genet, 15(5), 525-527, May. 2007.

[19] Vissers, L.E. and Stankiewicz, P., "Microdeletion and microduplication syndromes", Methods Mol Biol, 838, 29-75, 2012.

[20] Shaffer, L.G., Bejjani, B.A., Torchia, B., Kirkpatrick, S., Coppinger, J. and Ballif, B.C., "The identification of microdeletion syndromes and other chromosome abnormalities: cytogenetic methods of the past, new technologies for the future", Am J Med Genet C Semin Med Genet, 145C (4), 335-345, Nov. 2007.

[21] Li, M.M. and Andersson, H.C., "Clinical application of microarray-based molecular cytogenetics: an emerging new era of genomic medicine", J Pediatr, 155(3), 311-317, 2009.

[22] Kirchhoff, M., Bisgaard, A.M., Bryndorf, T. and Gerdes, T., "MLPA analysis for a panel of syndromes with mental retardation reveals imbalances in $5.8 \%$ of patients with mental ret ardation and dysmorphic feat ures, including duplications of the Sotos syndrome and Williams-Beuren syndrome regions", Eur J Med Genet, 50(1), 33-42, Jan-Feb. 2007

[23] Jehee, F.S., Takamori, J.T., Medeiros, P.F., Pordeus, A.C., Latini, F.R., Bertola, D.R., Kim, C.A. and Passos-Bueno, M.R., "Using a combination of MLPA kits to detect chromosomal imbalances in patients with multiple congenital anomalies and ment al retardation is a valuable choice for developing countries", Eur J Med Genet, 54(4), e425-432, Jul-Aug. 2011

[24] Schouten, J.P., McElgunn, C.J., Waaijer, R., et al. "Relative quantification of 40 nucleic acid sequences by multiplex ligationdependent probe amplification”, Nucleic Acids Res, 30(12), e57, Jun. 2002.

[25] Shaw-Smith, C., Redon, R., Rickman, L., et al. "Microarray based comparative genomic hybridisation (array-CGH) detects submicroscopic chromosomal deletions and duplications in patients with learning disability/mental retardation and dysmorphic feat ures", J Med Genet, 41(4), 241 -248, Apr. 2004.

[26] Krepischi-Santos, A.C., Vianna-Morgante, A.M., Jehee, F.S., et al. "Whole-genome array-CGH screening in undiagnosed syndromic patients: old syndromes revisited and new alterations", Cytogenet Genome Res, 115(3-4), 254-261, 2006.

[27] Bruno, D.L., Ganesamoorthy, D., Schoumans, J., et al. "Detection of cryptic pathogenic copy number variations and constitutional loss of heterozygosity using high resolution SNP microarray analysis in 117 patients referred for cytogenetic analysis and impact on clinical practice", J Med Genet, 46(2), 123-131, Feb. 2009

[28] Gijsbers, A.C., Lew, J.Y., Bosch, C.A., et al. "A new diagnostic workflow for patients with mental retardation and/or multiple congenital abnormalities: test arrays first", Eur J Hum Genet, 17(11), 1394-1402, Nov. 2009.

[29] Regier, D.A., Friedman, J.M. and Marra, C.A., "Value for money? Array genomic hybridization for diagnostic testing for genetic causes of intellectual disability", Am J Hum Genet, 86(5), 765-772, May 2010.

[30] Slavotinek, A.M. "Novel microdeletion syndromes detected by chromosome microarrays", Hum Genet, 124(1), 1-17, Aug. 2008.

[31] Vieira, T.P., Sgardioli, I.C. and Gil-da-Silva-Lopes, V.L. "Genetics and public health: the experience of a reference center for diagnosis of 22q1 1.2 deletion in Brazil and suggestions for implementing genet ic testing", J Community Genet, 4(1), 99-106, Jan. 2012.

[32] Programa das Nações Unidas para o Desenvolvimento do Brasil (PNUD Brasil). "Atlas do Desenvolvimento Humano no Brasil", 2002. [Accessed April 16, 2013].

[33] Pober, B.R., "Williams-Beuren syndrome", $N$ Engl J Med, 362(3), 239-252, Jan. 2010.

[34] McDonald-McGinn, D.M. and Sullivan, K.E., "Chromosome 22q1 1.2 deletion syndrome (DiGeorge syndrome/velocardiofacial syndrome)", Medicine (Baltimore), 90(1), 1-18, Jan. 2011.

[35] Paim, J., Travassos, C., Almeida, C., Bahia, L. and Macinko, J. "The Brazilian health system: history, advances, and challenges", Lancet, 377(9779), 1778-1797, May 2011.

[36] Horovitz, D.D., Cardoso, M.H., Llerena, J.C. Jr., de Mattos, R.A. "Birth defects in Brazil and health care: proposals for public policies in clinical genetics", Cad Saude Publica 22(12): 25992609, Dec. 2006.

[37] Castilla, E.E. and Luquetti, D.V., "Brazil: public health genomics", Public Health Genomics, 12(1), 53-58, 2009.

[38] Horovitz, D.D., de Faria Ferraz, V.E., Dain, S. and Marques-deFaria, A.P. "Genetic services and test ing in Brazil", J Community Genet, [Epub ahead of print], May 2012

[39] Melo, D.G. and Sequeiros, J., "The challenges of incorporating genetic testing in the unified national health system in Brazil", Genet Test Mol Biomarkers, 16(7), 651-655, Jul. 2012.

[40] Brasil, Ministério da Saúde, Gabinete do Ministro. "Portaria $N^{o} 81$, de 20 de janeiro de 2009 - Institui, no âmbito do SUS, a Política Nacional de Atenção Integral em Genética Clínica”, Diário Oficial da União da República Federativa do Brasil, Brasília, 21 de janeiro de 2009. [Accessed April 16, 2013].

[41] Scheffer, M., Biancarelli, A., Cassenote, A. (coords.), "Demografia Médica no Brasil: dados gerais e descrições de desigualdades", São Paulo: Conselho Regional de Medicina do Estado de São Paulo e Conselho Federal de Medicina, 2011. [Accessed April 16, 2013]. 